\title{
Resonant Integral Boundary Value Problems for Caputo Fractional Differential Equations
}

\author{
Wenjie Ma, ${ }^{1}$ Shuman Meng, ${ }^{1}$ and Yujun Cui $\mathbb{D}^{1,2}$ \\ ${ }^{1}$ Department of Applied Mathematics, Shandong University of Science and Technology, Qingdao 266590, China \\ ${ }^{2}$ State Key Laboratory of Mining Disaster Prevention and Control Cofounded by Shandong Province and \\ the Ministry of Science and Technology, Shandong University of Science and Technology, Qingdao 266590, China
}

Correspondence should be addressed to Yujun Cui; cyj720201@163.com

Received 3 May 2018; Accepted 30 July 2018; Published 7 August 2018

Academic Editor: Zhen-Lai Han

Copyright (C) 2018 Wenjie Ma et al. This is an open access article distributed under the Creative Commons Attribution License, which permits unrestricted use, distribution, and reproduction in any medium, provided the original work is properly cited.

This paper deals with the following Caputo fractional differential equations with Riemann-Stieltjes integral boundary conditions ${ }^{c} D_{0+}^{\alpha} u(t)=f\left(t, u(t), u^{\prime}(t), u^{\prime \prime}(t)\right), t \in[0,1], u(0)=u^{\prime \prime}(0)=0, u(1)=\int_{0}^{1} \mathrm{u}(t) d A(t)$, where ${ }^{c} D_{0+}^{\alpha}$ denotes the standard Caputo derivative, $\alpha \in(2,3]$; $\int_{0}^{1} x(t) d A(t)$ denotes the Riemann-Stieltjes integrals of $x$ with respect to $A$. By mean of coincidence degree theory, we obtain the existence of solutions for the above fractional BVP at resonance. In the end, according to the main results, we give a typical example.

\section{Introduction}

Fractional differential equations have various applications in chemical engineering, blood flow phenomena, physics, signal and image processing, fitting of experimental data, control theory, etc. Fractional differential equations also are a fundamental tool for the description of process of natural phenomena. In past years, many authors studied the existence of solutions or positive solutions of various fractional differential equations such as fractional boundary value problem at resonance [1-9], fractional boundary value problem at nonresonance [10-23], Caputo fractional derivative problem $[24,25]$, impulsive problem [26], conjugate boundary value problems $[27,28]$, integral boundary value problem [29], variational structure problem [30], fractional $p$-Laplace problem [31], fractional lower and upper solution problem $[32,33]$, solitons $[34,35]$, etc.

For example, Alberto et al. [11] considered the following fractional differential equations:

$$
\begin{aligned}
{ }^{c} D^{\alpha} u(t)+f(t, u(t)) & =0, \quad t \in(0,1), \\
u(0) & =u^{\prime \prime}(0)=0, \\
u(1) & =\lambda \int_{0}^{1} u(s) d s,
\end{aligned}
$$

where $\lambda \in(0,2), \alpha \in(2,3),{ }^{c} D_{0+}^{\alpha}$ denotes the Caputo fractional derivative, and $f:[0,1] \times[0, \infty) \longrightarrow[0, \infty)$ is a continuous function. The existence of solutions is obtained by using the Guo-Krasnoselskii fixed point theorem. Similar arguments for related issues are treated in [12].

In [7], by mean of coincidence degree theory, the existence of solution of integral BVP for nonlinear differential equation is proved:

$$
\begin{aligned}
-D_{0+}^{p} x(t) & =f\left(t, x(t), D_{0+}^{p-1} x(t), D_{0+}^{p-2} x(t)\right), \\
x(0) & =x^{\prime}(0)=0, \\
x(1) & =\int_{0}^{1} x(t) d A(t),
\end{aligned}
$$

where $p \in(2,3), D_{0+}^{p}$ is the Riemann-Liouville derivative, $f:[0,1] \times \mathbb{R}^{3} \longrightarrow \mathbb{R}$, and $f$ satisfies Caratheodory conditions. Similar method for different nonlinear differential equations, with either Caputo or Riemann-Liouville derivatives, and using suitable coincidence degree theory in each case, has allowed proving the existence of solutions in many papers; see also [1-5, 24, 25, 33]. 
In [5], the authors studied nonlocal fractional differential equation:

$$
\begin{aligned}
D_{0+}^{\alpha} u(t)+f(t, u(t)) & =0, \quad t \in(0,1), \\
u(0) & =0, \\
u(1) & =\eta u(\xi),
\end{aligned}
$$

where $\eta \xi^{\alpha-1}=1, \xi \in(0,1), \alpha \in(1,2), D_{0+}^{\alpha}$ is the Riemann-Liouville derivative, and $f:[0,1] \times[0, \infty) \longrightarrow R$ is continuous function. They solved the above differential equation by using iterative technique and the fixed point index theory. We can also refer to $[16,17]$.

In this paper, we are concerned with the existence of solution for the following differential equation with boundary value conditions:

$$
\begin{aligned}
{ }^{c} D_{0+}^{\alpha} u(t) & =f\left(t, u(t), u^{\prime}(t), u^{\prime \prime}(t)\right), \quad t \in[0,1], \\
u(0) & =u^{\prime \prime}(0)=0, \\
u(1) & =\int_{0}^{1} u(t) d A(t),
\end{aligned}
$$

where ${ }^{c} D_{0+}^{\alpha}$ denotes the Caputo fractional derivative, $\alpha \in$ $(2,3]$, and $f:[0,1] \times R^{3} \longrightarrow R$ is a continuous function. The $\operatorname{BVP}(4)$ is a resonance problem, because $\int_{0}^{1} t d A(t)=1$, if and only if its associated linear homogeneous BVP

$$
\begin{gathered}
{ }^{c} D_{0+}^{\alpha} x(t)=0, \quad t \in[0,1], \\
x(0)=x^{\prime \prime}(0)=0, \\
x(1)=\int_{0}^{1} x(t) d A(t)
\end{gathered}
$$

have nontrivial solution. Here, we assume that the following two conditions hold:

$$
\begin{aligned}
& \left(A_{1}\right) \int_{0}^{1} t d A(t)=1, \int_{0}^{1} t^{\alpha} d A(t) \neq 1 . \\
& \left(A_{2}\right) f:[0,1] \times R^{3} \longrightarrow R \text { is a continuous function. }
\end{aligned}
$$

As is known to all, there are few studies on such boundary value problems. Therefore, by mean of coincidence degree theory, we consider the existence of solutions for fractional differential equations (4) with Riemann-Stieltjes integral boundary conditions at resonance.

\section{Preliminaries}

For the convenience of the reading, we introduce the theorem and lemma of fractional calculus theory.

Lemma 1 (see [36]). Put $\alpha \in(0,+\infty), f \in L(0,1) \cap C(0,1)$; the fractional differential equation

$$
{ }^{c} D_{0+}^{\alpha} f(x)=0
$$

has the general solution $f(x)=A_{0}+A_{1} x+\cdots+A_{n-1} x^{n-1}$, $A_{k} \in R, k=0,1 \ldots, n-1$, where $n$ is the smallest integer greater than or equal to $\alpha$.
Lemma 2 (see [36]). Given that $f \in C^{n}[0,1]$, then

$$
\begin{aligned}
I_{0+}^{\alpha c} D_{0+}^{\alpha} f(x)= & f(x)-f(0)-f^{\prime}(0) x-\cdots \\
& -\frac{f^{(n-1)}(0)}{(n-1) !} x^{n-1}
\end{aligned}
$$

where $n$ is the smallest integer greater than or equal to $\alpha$.

Let $Y, Z$ be two Banach spaces and $L: D(L) \subset Y \longrightarrow Z$ be a linear operator. Assume that $L$ is a Fredholm operator of index zero; $P: Y \longrightarrow Y, Q: Z \longrightarrow Z$ are two continuous projectors such that

$$
\begin{aligned}
\operatorname{Im} P & =\operatorname{ker} L, \\
\operatorname{ker} Q & =\operatorname{Im} L, \\
Y & =\operatorname{ker} L \oplus \operatorname{ker} P, \\
Z & =\operatorname{Im} L \oplus \operatorname{Im} Q .
\end{aligned}
$$

Thus

$$
\left.L\right|_{\operatorname{Ker} P \cap D(L)}: \operatorname{Ker} P \cap D(L) \longrightarrow \operatorname{Im} L
$$

is invertible. We denote the inverse of the mapping by $K_{P}$ (generalized inverse operator of $L$ ). If $\Omega$ is an open bounded subset of $Y$ such that $D(L) \cap \Omega \neq \emptyset, N: Y \rightarrow Z$ will be called $L$-compact on $\bar{\Omega}$ if $Q N(\bar{\Omega})$ is bounded and $K_{P}(I-Q) N$ : $\bar{\Omega} \longrightarrow Y$ is compact.

Theorem 3 (see [37]). $L: D(L) \subset Y \longrightarrow Z$ is a linear operator, and $L$ is a Fredholm operator of index zero, and $N$ is $L$-compact on $\bar{\Omega}$. The equation $L \psi=N \psi$ has at least one solution in $D(L) \cap \bar{\Omega}$ if and only if the following three conditions are satisfied:

(1) $L \psi \neq \lambda N \psi$ for every $(\psi, \lambda) \in[(D(L) \backslash \operatorname{ker} L) \cap \partial \Omega] \times$ $(0,1)$.

(2) $N \psi \notin \operatorname{Im} L$ for every $\psi \in \operatorname{Ker} L \cap \partial \Omega$.

(3) $\operatorname{deg}\left(\left.Q N\right|_{\mathrm{Ker} L}, \Omega \cap \operatorname{Ker} L, 0\right) \neq 0$, where $Q: Y \longrightarrow Y$ is a projection such that $\operatorname{Im} L=\operatorname{Ker} Q$.

In this paper, let $Y=C^{2}[0,1]$ with the norm $\|x\|=$ $\max \left\{\|x\|_{\infty},\left\|x^{\prime}\right\|_{\infty},\left\|x^{\prime \prime}\right\|_{\infty}\right\}$ in which $\|x\|_{\infty}=\max _{t \in[0,1]}|x(t)|$. Let $Z=C[0,1]$ with the norm $\|x\|_{\infty}=\max _{t \in[0,1]}|x(t)|$. Operator $L$ is defined as $L: L u(t)={ }^{c} D_{0+}^{\alpha} u(t)$ with

$$
\begin{aligned}
& D(L)=\left\{u \in Y:{ }^{c} D_{0+}^{\alpha} u(t) \in C[0,1], u(0)=u^{\prime \prime}(0)\right. \\
& \left.=0, u(1)=\int_{0}^{1} u(t) d A(t)\right\} .
\end{aligned}
$$

Define operator $N: Y \longrightarrow Z$ as follows:

$$
(N u)(x)=f\left(t, u(t), u^{\prime}(t), u^{\prime \prime}(t)\right) .
$$

And so problem (4) becomes $L u=N u, u \in D(L)$. 


\section{Main Results}

In this section, we continue to give the following assumptions.

$\left(A_{3}\right)$ There exist functions $a(t), b(t), c(t), d(t) \in C[0,1]$ with $\|b\|_{\infty}+\|c\|_{\infty}+\|d\|_{\infty}<\Gamma(\alpha-1)$ such that

$$
\begin{array}{r}
|f(t, u, v, w)| \leq a(t)+b(t)|u|+c(t)|v|+d(t)|w|, \\
t \in[0,1] .
\end{array}
$$

$\left(A_{4}\right)$ There exists positive constant $F$ such that, for $y \in$ Dom $L$, if $\left|y^{\prime}(t)\right|>F$ for $t \in[0,1]$, then

$$
\begin{aligned}
\int_{0}^{1}(1-s)^{\alpha-1}(N y)(s) d s \\
\quad-\int_{0}^{1} \int_{0}^{t}(t-s)^{\alpha-1}(N y)(s) d s d A(t) \neq 0 .
\end{aligned}
$$

$\left(A_{5}\right)$ There exists constant $a_{0}>0$ such that either

$$
\begin{aligned}
& c\left[\int_{0}^{1}(1-s)^{\alpha-1} f(s, c s, c, 0) d s\right. \\
& \left.\quad-\int_{0}^{1} \int_{0}^{t}(t-s)^{\alpha-1} f(s, c s, c, 0) d s d A(t)\right]<0
\end{aligned}
$$

or

$$
\begin{aligned}
& c\left[\int_{0}^{1}(1-s)^{\alpha-1} f(s, c s, c, 0) d s\right. \\
& \left.\quad-\int_{0}^{1} \int_{0}^{t}(t-s)^{\alpha-1} f(s, c s, c, 0) d s d A(t)\right]>0
\end{aligned}
$$

holds if $|c|>a_{0}$.

Based on the above, we will prove the following important theorem.

Theorem 4. Suppose that $\left(A_{1}\right)-\left(A_{5}\right)$ is satisfied; there exists at least one solution of the fractional differential equation (4) in $Y$.

For convenience, we first give some important lemmas as follows.

Lemma 5. Assume that $\left(A_{1}\right)$ is satisfied; then $L: D(L) \subset$ $Y \longrightarrow Z$ is Fredholm operator with index zero, and a linear continuous projector $Q: Z \longrightarrow Z$ is defined by

$$
\begin{aligned}
& (Q y)(t)=\frac{\alpha}{1-\int_{0}^{1} t^{\alpha} d A(t)}\left[\int_{0}^{1}(1-s)^{\alpha-1} y(s) d s\right. \\
& \left.-\int_{0}^{1} \int_{0}^{t}(t-s)^{\alpha-1} y(s) d s d A(t)\right] .
\end{aligned}
$$

Furthermore, define a linear operator $K_{p}: \operatorname{Im} L \longrightarrow D(L) \cap$ ker $P$ as follows:

$$
\begin{aligned}
\left(K_{p} y\right)(t)= & \frac{1}{\Gamma(\alpha)} \int_{0}^{t}(t-s)^{\alpha-1} y(s) d s \\
& -\frac{t}{\Gamma(\alpha)} \int_{0}^{1}(1-s)^{\alpha-1} y(s) d s
\end{aligned}
$$

such that $K_{p}=\left(\left.L\right|_{D(L) \cap \mathrm{ker} P}\right)^{-1}$.
Proof. It follows from Lemma 1 and $L u=0$ that

$$
u(t)=c_{1}+c_{2} t+c_{3} t^{2}, \quad c_{1}, c_{2}, c_{3} \in R .
$$

According to $u(0)=u^{\prime \prime}(0)=0, u(1)=\int_{0}^{1} u(t) d A(t)$, we have

$$
\operatorname{ker} L=\{c t: c \in R\} .
$$

Moreover, we can obtain that

$$
\begin{aligned}
\operatorname{Im} & L=\left\{y \in Z: \int_{0}^{1}(1-s)^{\alpha-1} y(s) d s\right. \\
& \left.-\int_{0}^{1} \int_{0}^{t}(t-s)^{\alpha-1} y(s) d s d A(t)=0\right\} .
\end{aligned}
$$

On one hand, suppose $y \in \operatorname{Im} L$; then there exist $u \in D(L)$ :

$$
y=L u \in Z \text {. }
$$

Then we have

$$
u(t)=I_{0+}^{\alpha} y(t)+c_{1}+c_{2} t+c_{3} t^{2}
$$

This together with $\left(A_{1}\right), u(0)=u^{\prime \prime}(0)=0, u(1)=$ $\int_{0}^{1} u(t) d A(t)$, we can get $c_{1}=c_{3}=0$ and

$$
\begin{aligned}
& \int_{0}^{1}(1-s)^{\alpha-1} y(s) d s-\int_{0}^{1} \int_{0}^{t}(t-s)^{\alpha-1} y(s) d s d A(t) \\
& \quad=0 .
\end{aligned}
$$

And so we obtain that

$$
\begin{aligned}
\operatorname{Im} & L \subset\left\{y \in Z: \int_{0}^{1}(1-s)^{\alpha-1} y(s) d s\right. \\
- & \left.\int_{0}^{1} \int_{0}^{t}(t-s)^{\alpha-1} y(s) d s d A(t)=0\right\} .
\end{aligned}
$$

On the other hand, if $y \in Z$ satisfies

$$
\begin{aligned}
& \int_{0}^{1}(1-s)^{\alpha-1} y(s) d s-\int_{0}^{1} \int_{0}^{t}(t-s)^{\alpha-1} y(s) d s d A(t) \\
& \quad=0
\end{aligned}
$$

we let

$$
\begin{aligned}
u(t)= & I_{0+}^{\alpha} y(t)-t I_{0+}^{\alpha} y(1) \\
= & \frac{1}{\Gamma(\alpha)} \int_{0}^{t}(1-s)^{\alpha-1} y(s) d s \\
& -\frac{t}{\Gamma(\alpha)} \int_{0}^{1}(1-s)^{\alpha-1} y(s) d s .
\end{aligned}
$$

Then we conclude that

$$
\begin{aligned}
& u(0)=u^{\prime \prime}(0)=0, \\
& u(1)=I_{0+}^{\alpha} y(1)-\frac{1}{\Gamma(\alpha)} \int_{0}^{1}(1-s)^{\alpha-1} y(s) d s=0
\end{aligned}
$$


and

$$
\begin{aligned}
& \int_{0}^{1} u(t) d A(t) \\
& =\int_{0}^{1} \frac{1}{\Gamma(\alpha)} \int_{0}^{t}(1-s)^{\alpha-1} y(s) d s d A(t) \\
& \quad-\int_{0}^{1} t d A(t) \frac{1}{\Gamma(\alpha)} \int_{0}^{1}(1-s)^{\alpha-1} y(s) d s \\
& =\int_{0}^{1} \frac{1}{\Gamma(\alpha)} \int_{0}^{t}(1-s)^{\alpha-1} y(s) d s d \mathrm{~A}(t) \\
& \quad-\frac{1}{\Gamma(\alpha)} \int_{0}^{1}(1-s)^{\alpha-1} y(s) d s=0 .
\end{aligned}
$$

That is, $u \in D(L)$, then $y \in \operatorname{Im} L$. In conclusion,

$$
\begin{aligned}
\operatorname{Im} L & =\left\{y \in Z: \int_{0}^{1}(1-s)^{\alpha-1} y(s) d s\right. \\
& \left.-\int_{0}^{1} \int_{0}^{t}(t-s)^{\alpha-1} y(s) d s d A(t)=0\right\} .
\end{aligned}
$$

We give projection operator $P: Y \longrightarrow Y$ as

$$
(P u)(t)=u(1) t .
$$

We get Ker $P=\{u \in Y \mid u(1)=0\}, P^{2} u=P u$, and $\operatorname{Im} P=$ ker $L$. For any $u \in Y$, together with $u=(u-P u)+P u$, we have $Y=\operatorname{ker} P+\operatorname{ker} L$. It is easy to obtain that $\operatorname{ker} L \cap \operatorname{ker} P=\{0\}$ which implies

$$
Y=\operatorname{ker} P \oplus \operatorname{ker} L
$$

Next operator $Q: Z \longrightarrow Z$ is defined as follows:

$$
\begin{gathered}
(\mathrm{Q} y)(t)=\frac{\alpha}{1-\int_{0}^{1} t^{\alpha} d A(t)}\left[\int_{0}^{1}(1-s)^{\alpha-1} y(s) d s\right. \\
\left.-\int_{0}^{1} \int_{0}^{t}(t-s)^{\alpha-1} y(s) d s d A(t)\right] .
\end{gathered}
$$

Noting that

$$
\begin{aligned}
& \left(Q^{2} y\right)(t)=(Q(Q y))(t) \\
& =\frac{\alpha}{1-\int_{0}^{1} t^{\alpha} d A(t)}\left[\int_{0}^{1}(1-s)^{\alpha-1} Q y(s) d s\right. \\
& \left.-\int_{0}^{1} \int_{0}^{t}(t-s)^{\alpha-1} Q y(s) d s d A(t)\right]=Q y(t) \\
& \cdot \frac{\alpha}{1-\int_{0}^{1} t^{\alpha} d A(t)}\left[\int_{0}^{1}(1-s)^{\alpha-1} d s\right. \\
& \left.-\int_{0}^{1} \int_{0}^{t}(t-s)^{\alpha-1} d s d A(t)\right]=(Q y)(t)
\end{aligned}
$$

means $Q$ is a projection operator. And obviously, $\operatorname{ker} Q=$ $\operatorname{Im} L$. For any $y \in Z$, let $y_{1}=y-Q y$,

$$
\begin{aligned}
& \left(\mathrm{Q} y_{1}\right)(t)=(\mathrm{Q}(y-\mathrm{Q} y))(t) \\
& =\frac{\alpha}{1-\int_{0}^{1} t^{\alpha} d A(t)}\left[\int_{0}^{1}(1-s)^{\alpha-1}\right. \\
& \cdot(y(s)-\mathrm{Q} y(s)) d s \\
& \left.-\int_{0}^{1} \int_{0}^{t}(t-s)^{\alpha-1}(y(s)-Q y(s)) d s d A(t)\right] \\
& =\frac{\alpha}{1-\int_{0}^{1} t^{\alpha} d A(t)}\left[\int_{0}^{1}(1-s)^{\alpha-1} y(s) d s\right. \\
& \left.-\int_{0}^{1} \int_{0}^{t}(t-s)^{\alpha-1} y(s) d s d A(t)\right] \\
& +\frac{\alpha}{1-\int_{0}^{1} t^{\alpha} d A(t)}\left[\int_{0}^{1}(1-s)^{\alpha-1} Q y(s) d s\right. \\
& \left.-\int_{0}^{1} \int_{0}^{t}(t-s)^{\alpha-1} Q y(s) d s d A(t)\right]=(Q y)(t) \\
& -\left(Q^{2} y\right)(t)=0,
\end{aligned}
$$

and we have $y_{1} \in \operatorname{Im} L$, so $Z=\operatorname{Im} L+\operatorname{Im} Q$. Moreover, by simple calculation, we can get $\operatorname{Im} Q \cap \operatorname{Im} L=\{0\}$. Above all, $Z=\operatorname{Im} L \oplus \operatorname{Im} Q$.

To summarize, we can know that $\operatorname{Im} L$ is closed subspace of $Z$; $\operatorname{dimker} L=\operatorname{codim} \operatorname{Im} L=1$; and so $L$ is a Fredholm operator of index zero.

Next we will prove that $K_{p}$ is the inverse of $\left.L\right|_{\text {dom } n \text { ker } P}$. It is clear that

$$
\left(L_{p} K_{p} y\right)(t)={ }^{c} D_{0+}^{\alpha} K_{p} y(t)=y(t), \quad y \in \operatorname{Im} L .
$$

For each $u \in \operatorname{dom} L \cap \operatorname{ker} P$, we have $u(0)=u^{\prime \prime}(0)=0, u(1)=$ 0 and

$$
\begin{aligned}
\left(K_{p} L_{p} u\right)(t) & =I_{0+}^{\alpha c} D_{0+}^{\alpha} u(t)-t I_{0+}^{\alpha c} D_{0+}^{\alpha}(1) \\
& =u(t)+u^{\prime}(0) t-t\left(u(1)+u^{\prime}(0)\right) \\
& =u(t) .
\end{aligned}
$$

This implies that $K_{p} L_{p} u=u$. So $K_{p}=\left(\left.L\right|_{\text {domLnker } P}\right)^{-1}$.

Lemma 6. Assume that $\Omega$ is a bounded open subset of $Y$, then $N$ is L-compact on $\bar{\Omega}$ if domL $\cap \bar{\Omega} \neq \emptyset$.

Proof. By the continuity of $f$, we obtain $Q N(\bar{\Omega})$ and (I Q) $N(\bar{\Omega})$ are bounded, so there exists constant $M>0$, such that $|(I-Q) N u(t)| \leq M$ for $u \in \bar{\Omega}$ and $t \in[0,1] . \forall t_{1}, t_{2} \in$ 
$[0,1], t_{1}<t_{2}, u \in \bar{\Omega}$, let $(O u)(s)=(I-Q) N u(s)$, and we have

$$
\begin{aligned}
& \left|\left(K_{P}(I-Q) N u\right)\left(t_{2}\right)-\left(K_{P}(I-Q) N u\right)\left(t_{1}\right)\right| \\
& \quad \leq \frac{1}{\Gamma(\alpha)} \mid \int_{0}^{t_{2}}\left(t_{2}-s\right)^{\alpha-1}(\mathrm{Ou})(s) d s \\
& \quad-\int_{0}^{t_{1}}\left(t_{1}-s\right)^{\alpha-1} \mathrm{Ou}(s) d s \mid \\
& \quad+\frac{t_{2}-t_{1}}{\Gamma(\alpha)}\left|\int_{0}^{1}(1-s)^{\alpha-1}(\mathrm{Ou})(s) d s\right| \\
& \quad \leq \frac{M}{\Gamma(\alpha)} \mid \int_{0}^{t_{1}}\left[\left(t_{2}-s\right)^{\alpha-1}-\left(t_{1}-s\right)^{\alpha-1}\right] d s \\
& \quad+\int_{t_{1}}^{t_{2}}\left(t_{2}-s\right)^{\alpha-1} d s \mid+\frac{M\left(t_{2}-t_{1}\right)}{\Gamma(\alpha)} \int_{0}^{1}(1-s)^{\alpha-1} d s \\
& \quad=\frac{M}{\Gamma(\alpha+1)}\left(t_{2}^{\alpha}-t_{1}^{\alpha}+t_{2}-t_{1}\right), \\
& \quad+\int_{t_{1}}^{t_{2}}\left(t_{2}-s\right)^{\alpha-2} d s \mid=\frac{M}{\Gamma(\alpha)}\left(t_{2}^{\alpha-1}-t_{1}^{\alpha-1}\right), \\
& \quad=\frac{M}{\Gamma(\alpha-1)} \mid \int_{0}^{t_{1}}\left[\left(t_{2}-s\right)^{\alpha-2}-\left(t_{1}-s\right)^{\alpha-2}\right] d s \\
& \quad-\int_{0}^{t_{1}}\left(t_{1}-s\right)^{\alpha-2} \mathrm{Ou}(s) d s \mid \\
& \quad \leq \int_{0}^{t_{2}}\left(t_{2}-s\right)^{\alpha-2}(\mathrm{Ou})(s) d s
\end{aligned}
$$

and

$$
\begin{aligned}
& \left|\left(K_{P}(I-Q) N u\right)^{\prime \prime}\left(t_{2}\right)-\left(K_{P}(I-Q) N u\right)^{\prime \prime}\left(t_{1}\right)\right| \\
& \quad=\frac{1}{\Gamma(\alpha-2)} \mid \int_{0}^{t_{2}}\left(t_{2}-s\right)^{\alpha-3}(\mathrm{Ou})(s) d s \\
& \quad-\int_{0}^{t_{1}}\left(t_{1}-s\right)^{\alpha-3} \mathrm{Ou}(s) d s \mid \\
& \quad \leq \frac{M}{\Gamma(\alpha-2)} \mid \int_{0}^{t_{1}}\left[\left(t_{2}-s\right)^{\alpha-3}-\left(t_{1}-s\right)^{\alpha-3}\right] d s \\
& \quad+\int_{t_{1}}^{t_{2}}\left(t_{2}-s\right)^{\alpha-3} d s \mid=\frac{M}{\Gamma(\alpha-1)}\left(t_{2}^{\alpha-2}-t_{1}^{\alpha-2}\right) .
\end{aligned}
$$

As a result of $t^{\alpha}, t^{\alpha-1}, t^{\alpha-2}$ are uniformly continuous on $[0,1]$. So we have that $K_{P}(I-Q) N(\bar{\Omega}) \subset C[0,1],\left(K_{P}(I-\right.$ $Q) N)^{\prime}(\bar{\Omega}) \subset C[0,1],\left(K_{P}(I-Q) N\right)^{\prime \prime}(\bar{\Omega}) \subset C[0,1]$ are equicontinuous, and, by Ascoli-Arzela theorem, we have that $K_{P}(I-Q) N: \bar{\Omega} \longrightarrow Y$ is compact. Thus, $N$ is $L$-compact.
Lemma 7. Set $\Omega_{1}=\{u \in \operatorname{ker} L: \lambda u+\beta(1-\lambda) Q N u=$ $0, \lambda \in[0,1]\}$ is bounded if conditions $\left(A_{1}\right),\left(A_{2}\right)$, and $\left(A_{5}\right)$ are satisfied, and

$$
\beta= \begin{cases}-1, & \text { if (14) holds } \\ 1, & \text { if }(15) \text { holds } .\end{cases}
$$

Proof. Suppose that $u \in \Omega_{1}$ we have $u(t)=c t, c \in R$ and

$$
\begin{aligned}
\lambda c t & +\beta(1-\lambda) \frac{\alpha}{1-\int_{0}^{1} t^{\alpha} d A(t)} \\
\cdot & {\left[\int_{0}^{1}(1-s)^{\alpha-1} f(s, c s, c, 0) d s\right.} \\
- & \left.\int_{0}^{1} \int_{0}^{t}(t-s)^{\alpha-1} f(s, c s, c, 0) d s d A(t)\right]=0
\end{aligned}
$$

If $\lambda=0$, by condition $\left(A_{5}\right)$, we have $|c| \leq a_{0}$. If $\lambda=1$, then $c=0$. If $\lambda \in(0,1)$, we suppose $|c| \geq a_{0}$, then

$$
\begin{aligned}
& \lambda c^{2} t=-\beta(1-\lambda) c \frac{\alpha}{1-\int_{0}^{1} t^{\alpha} d A(t)} \\
& \cdot\left[\int_{0}^{1}(1-s)^{\alpha-1} f(s, c s, c, 0) d s\right. \\
& \left.-\int_{0}^{1} \int_{0}^{t}(t-s)^{\alpha-1} f(s, c s, c, 0) d s d A(t)\right]<0
\end{aligned}
$$

which contradicts with $\lambda c^{2}>0$.

Lemma 8. Set $\Omega_{2}=\{u \in D(L) \backslash \operatorname{ker} L: L u=\lambda N u, \lambda \in$ $[0,1]\}$ is bounded if $\left(A_{1}\right)-\left(A_{4}\right)$ are satisfied.

Proof. Take $u \in \Omega_{2}$, then $N u \in \operatorname{Im} L$. Thus we have

$$
\begin{aligned}
\int_{0}^{1}(1 & -s)^{\alpha-1}(N y)(s) d s \\
& -\int_{0}^{1} \int_{0}^{t}(t-s)^{\alpha-1}(N y)(s) d s d A(t)=0 .
\end{aligned}
$$

According to $\left(A_{4}\right)$, there exists constant $\xi \in(0,1)$ such that

$$
\left|u^{\prime}(\xi)\right| \leq F
$$

Because $\left|u^{\prime}(t)\right| \leq\left|u^{\prime}(\xi)\right|+\left|\int_{\xi}^{t} u^{\prime \prime}(s) d s\right| \leq F+\left\|u^{\prime \prime}\right\|_{\infty}$,

$$
\left\|u^{\prime}\right\|_{\infty} \leq F+\left\|u^{\prime \prime}\right\|_{\infty} .
$$

If $u \in D(L)$, we have $u(0)=0$, then

$$
|u(t)|=\left|u(0)+\int_{0}^{t} u^{\prime}(s) d s\right| \leq\left\|u^{\prime}\right\|_{\infty},
$$

and therefore $\|u\|_{\infty} \leq\left\|u^{\prime}\right\|_{\infty}$. 
By $L u=\lambda N u$, we have

$u(t)$

$$
\begin{aligned}
= & \frac{\lambda}{\Gamma(\alpha)} \int_{0}^{t}(t-s)^{\alpha-1} f\left(s, u(s), u^{\prime}(s), u^{\prime \prime}(s)\right) d s \\
& -u(0)-u^{\prime}(0) t-\frac{u^{\prime \prime}(0)}{2} t^{2} .
\end{aligned}
$$

Then we obtain that

$$
\begin{aligned}
u^{\prime \prime}(t) & \\
= & \frac{\lambda}{\Gamma(\alpha-2)} \int_{0}^{t}(t-s)^{\alpha-3} f\left(s, u(s), u^{\prime}(s), u^{\prime \prime}(s)\right) d s \\
& -u^{\prime \prime}(0) .
\end{aligned}
$$

According to the boundary condition $u^{\prime \prime}(0)=0$, one has

$$
\begin{aligned}
& u^{\prime \prime}(t)=\frac{\lambda}{\Gamma(\alpha-2)} \\
& \cdot \int_{0}^{t}(t-s)^{\alpha-3} f\left(s, u(s), u^{\prime}(s), u^{\prime \prime}(s)\right) d s .
\end{aligned}
$$

So we get

$$
\begin{aligned}
& \left|u^{\prime \prime}(t)\right| \leq \frac{1}{\Gamma(\alpha-2)} \int_{0}^{t}(t-s)^{\alpha-3} \\
& \cdot\left|f\left(s, u(s), u^{\prime}(s), u^{\prime \prime}(s)\right)\right| d s \leq \frac{1}{\Gamma(\alpha-2)} \\
& \quad \cdot \int_{0}^{1}(1-s)^{\alpha-3}\left|f\left(s, u(s), u^{\prime}(s), u^{\prime \prime}(s)\right)\right| d s \\
& \quad \leq \frac{1}{\Gamma(\alpha-2)} \int_{0}^{1}(1-s)^{\alpha-3}(a(s)+b(s)|u(s)| \\
& \left.\quad+c(s)\left|u^{\prime}(s)\right|+d(s)\left|u^{\prime \prime}(s)\right|\right) d s \\
& \quad \leq \frac{1}{\Gamma(\alpha-1)}\left(\|a\|_{\infty}+\|b\|_{\infty}\|u\|_{\infty}+\|c\|_{\infty}\left\|u^{\prime}\right\|_{\infty}\right. \\
& \left.+\|d\|_{\infty}\left\|u^{\prime \prime}\right\| \|_{\infty}\right) \leq \frac{1}{\Gamma(\alpha-1)}\left(\|a\|_{\infty}+\|b\|_{\infty}(F\right. \\
& \left.\left.+\left\|u^{\prime \prime}\right\|_{\infty}\right)+\|c\|_{\infty}\left(F+\left\|u^{\prime \prime}\right\| \|_{\infty}\right)+\|d\|_{\infty}\left\|u^{\prime \prime}\right\|_{\infty}\right) \\
& \quad \leq \frac{1}{\Gamma(\alpha-1)}\left(\|a\|_{\infty}+\|b\|_{\infty} F+\|c\|_{\infty} F+\left(\|b\|_{\infty}\right.\right. \\
& \left.\left.+\|c\|_{\infty}+\|d\|_{\infty}\right)\left\|u^{\prime \prime}\right\|_{\infty}\right) \cdot
\end{aligned}
$$

Thus, from $\|b\|_{\infty}+\|c\|_{\infty}+\|d\|_{\infty}<\Gamma(\alpha-1)$, we obtain that

$$
\left\|u^{\prime \prime}\right\|_{\infty} \leq \frac{\|a\|_{\infty}+F\left[\|b\|_{\infty}+\|c\|_{\infty}\right]}{\Gamma(\alpha-1)-\|b\|_{\infty}-\|c\|_{\infty}-\|d\|_{\infty}}=M_{1} .
$$

So we have

$$
\|u\|=\max \left\{\|u\|_{\infty},\left\|u^{\prime}\right\|_{\infty},\left\|u^{\prime \prime}\right\|_{\infty}\right\} \leq M_{1}+F .
$$

Therefore, $\Omega_{2}$ is bounded.
Lemma 9. Set $\Omega_{3}=\{u: u \in \operatorname{ker} L, N u \in \operatorname{Im} L\}$ is bounded if $\left(A_{1}\right),\left(A_{2}\right)$, and $\left(A_{5}\right)$ hold.

Proof. Let $u \in \Omega_{3}$, then $u(t)=c t, c \in R, N u \in \operatorname{Im} L$, and we can get

$$
\begin{aligned}
& \int_{0}^{1}(1-s)^{\alpha-1} f(s, c s, c, 0) d s \\
& \quad-\int_{0}^{1} \int_{0}^{t}(t-s)^{\alpha-1} f(s, c s, c, 0) d s d A(t)=0 .
\end{aligned}
$$

According to $\left(A_{5}\right)$ we have $|c| \leq a_{0}$, that is to say, $\Omega_{3}$ is bounded.

In summary, we can prove Theorem 4.

Proof of Theorem 4. Assume that $\Omega \supset \bigcup_{i=1}^{3} \overline{\Omega_{i}} \cup\{0\}$ is bounded open subset of $X$, and, according to Lemma 6 , we get that $N$ is $L$-compact on $\bar{\Omega}$. Form Lemmas 8 and 9, we have

(1) $L u \neq \lambda N u$, for all $(u, \lambda) \in[(D(L) \backslash \operatorname{ker} L) \cap \partial \Omega] \times$ $(0,1)$;

(2) $N u \notin \operatorname{Im} L$, for all $u \in \operatorname{Ker} L \cap \partial \Omega$.

Set $H(u, \lambda)=\lambda u+\beta(1-\lambda) Q N u$. It follows from Lemma 7 that we get $H(u, \lambda) \neq 0, u \in \partial \Omega \cap \operatorname{Ker} L$, so we have

$$
\begin{aligned}
& \operatorname{deg}\left(\left.Q N\right|_{\text {KerL }}, \Omega \cap \operatorname{Ker} L, 0\right)=\operatorname{deg}(\beta J, \Omega \cap \operatorname{Ker} L, 0) \\
& \quad \neq 0 .
\end{aligned}
$$

According to the above proof and Theorem 3, Theorem 4 holds.

Example 10. Considering the following fractional differential equation,

$$
\begin{aligned}
{ }^{c} D_{0+}^{5 / 2} u(t)= & t^{2}+t \frac{u^{2}(t)}{1+u^{2}(t)}+\frac{1}{4 \pi} u^{\prime}(t) \arctan u^{\prime}(t) \\
& +\frac{1}{4} \sin u^{\prime \prime}(t), \quad t \in[0,1], \\
u(0)= & u^{\prime \prime}(0)=0, \\
u(1)= & 2 \int_{0}^{1} u(t) d t .
\end{aligned}
$$

Obviously, $\alpha=5 / 2, A(t)=2 t$, then $\int_{0}^{1} t d A(t)=1$, and thus it is at resonance. Let

$$
f(t, u, v, w)=t^{2}+t \frac{u^{2}}{1+u^{2}}+\frac{1}{4 \pi} v \arctan v+\frac{1}{4} \sin w,
$$

then

$$
|f(t, u, v, w)| \leq 1+t^{2}+\frac{1}{2}|v|+\frac{1}{4}|w|
$$

where

$$
\|c\|_{\infty}+\|d\|_{\infty}=\frac{1}{2}+\frac{1}{4}=\frac{3}{4}<\frac{\sqrt{\pi}}{2}=\Gamma\left(\frac{3}{2}\right) .
$$


Taking $F=16$, then we have $\left|u^{\prime}(t)\right| \geq 16$,

$$
f\left(t, u(t), u^{\prime}(t), u^{\prime \prime}(t)\right) \geq-\frac{1}{2}+\frac{4}{\pi} \arctan 1-\frac{1}{4}>0 .
$$

and

$$
\begin{aligned}
\int_{0}^{1} & (1-s)^{\alpha-1}(N u)(s) d s \\
& -\int_{0}^{1} \int_{0}^{t}(t-s)^{\alpha-1}(N u)(s) d s d A(t) \\
& =\int_{0}^{1}(1-s)^{\alpha-1}(N u)(s) d s \\
& -2 \int_{0}^{1} \int_{s}^{1}(t-s)^{\alpha-1} d t(N u)(s) d s \\
& =\int_{0}^{1}\left((1-s)^{\alpha-1}-\frac{2(1-s)^{\alpha}}{\alpha}\right)(N u)(s) d s \\
& =\int_{0}^{1}(1-s)^{\alpha-1} \\
& . \frac{\alpha-2+2 s}{\alpha} f\left(s, u(s), u^{\prime}(s), u^{\prime \prime}(s)\right) d s>0 .
\end{aligned}
$$

That is, $\left(A_{4}\right)$ holds. Finally, taking $a_{0}=16$, when $|c|>16$, we conclude that

$$
\begin{aligned}
& \int_{0}^{1}(1-s)^{\alpha-1} f(s, c s, c, 0) d s \\
& \quad-\int_{0}^{1} \int_{0}^{t}(t-s)^{\alpha-1} f(s, c s, c, 0) d s d A(t)>0
\end{aligned}
$$

then $\left(A_{5}\right)$ is satisfied. It follows from Theorem 4 that (54) has at least one solution.

\section{Data Availability}

The data used to support the findings of this study are included within the article.

\section{Conflicts of Interest}

The authors declare that they have no conflicts of interest.

\section{Acknowledgments}

The research is supported by the National Natural Science Foundation (NNSF) of China (11371221 and 11571207), Shandong Natural Science Foundation (SDNSF) (ZR2018MA011), and the Tai'shan Scholar Engineering Construction Fund of Shandong Province of China.

\section{References}

[1] Z. Bai, "On solutions of some fractional m-point boundary value problems at resonance," Electronic Journal of Qualitative Theory of Differential Equations, vol. 37, pp. 1-15, 2010.
[2] W. Jiang, "Solvability for a coupled system of fractional differential equations at resonance," Nonlinear Analysis: Real World Applications, vol. 13, no. 5, pp. 2285-2292, 2012.

[3] W. Jiang, "The existence of solutions to boundary value problems of fractional differential equations at resonance," Nonlinear Analysis: Theory, Methods \& Applications, vol. 74, no. 5, pp. 1987-1994, 2011.

[4] N. Kosmatov, "A boundary value problem of fractional order at resonance," Electronic Journal of Differential Equations, vol. 135, pp. 1-10, 2010.

[5] Y. Wang and L. Liu, "Positive solutions for a class of fractional 3-point boundary value problems at resonance," Advances in Difference Equations, vol. 2017, article no. 7, 2017.

[6] Y. Zhang, Z. Bai, and T. Feng, "Existence results for a coupled system of nonlinear fractional three-point boundary value problems at resonance," Computers \& Mathematics with Applications, vol. 61, no. 4, pp. 1032-1047, 2011.

[7] Yumei Zou and Guoping He, "The existence of solutions to integral boundary value problems of fractional differential equations at resonance," Journal of Function Spaces, vol. 2017, Article ID 2785937, 7 pages, 2017.

[8] Y. Zou, L. Liu, and Y. Cui, "The Existence of Solutions for Four-Point Coupled Boundary Value Problems of Fractional Differential Equations at Resonance," Abstract and Applied Analysis, vol. 2014, Article ID 314083, 8 pages, 2014.

[9] Y. Zou and Y. Cui, "Existence results for a functional boundary value problem of fractional differential equations," Advances in Difference Equations, vol. 2013, no. 233, 2013.

[10] D. Băleanu, O. G. Mustafa, and R. P. Agarwal, "On $L^{P}$-solutions for a class of sequential fractional differential equations," Applied Mathematics and Computation, vol. 218, no. 5, pp. 20742081, 2011.

[11] A. Cabada and G. Wang, "Positive solutions of nonlinear fractional differential equations with integral boundary value conditions," Journal of Mathematical Analysis and Applications, vol. 212, pp. 403-411, 2012.

[12] A. Cabada and Z. Hamdi, "Nonlinear fractional differential equations with integral boundary value conditions," Applied Mathematics and Computation, vol. 228, pp. 251-257, 2014.

[13] Y. Cui, "Uniqueness of solution for boundary value problems for fractional differential equations," Applied Mathematics Letters, vol. 51, pp. 48-54, 2016.

[14] Y. Cui, W. Ma, Q. Sun, and X. Su, "New uniqueness results for boundary value problem of fractional differential equation," Nonlinear Analysis: Modelling and Control, vol. 23, no. 1, pp. 3139, 2018.

[15] Y. Cui, W. Ma, X. Wang, and X. Su, "Uniqueness theorem of differential system with coupled integral boundary conditions," Electronic Journal of Qualitative Theory of Differential Equations, vol. 2018, no. 9, pp. 1-10, 2018.

[16] J. Jiang, L. Liu, and Y. Wu, "Symmetric positive solutions to singular system with multi-point coupled boundary conditions," Applied Mathematics and Computation, vol. 220, pp. 536-548, 2013.

[17] J. Jiang, L. Liu, and Y. Wu, "Positive solutions to singular fractional differential system with coupled boundary conditions," Communications in Nonlinear Science and Numerical Simulation, vol. 18, no. 11, pp. 3061-3074, 2013.

[18] X. Leng, T. Feng, and X. Meng, "Stochastic inequalities and applications to dynamics analysis of a novel SIVS epidemic model with jumps," Journal of Inequalities and Applications, vol. 2017, article no. 138, 2017. 
[19] Qiao Sun, Hongwei Ji, and Yujun Cui, "Positive Solutions for Boundary Value Problems of Fractional Differential Equation with Integral Boundary Conditions," Journal of Function Spaces, vol. 2018, Article ID 6461930, 6 pages, 2018.

[20] X. G. Zhang, L. S. Liu, Y. H. Wu, and B. Wiwatanapataphee, “The spectral analysis for a singular fractional differential equation with a signed measure," Applied Mathematics and Computation, vol. 257, pp. 252-263, 2015.

[21] X. Zhang, L. Liu, and Y. Wu, “The eigenvalue problem for a singular higher order fractional differential equation involving fractional derivatives," Applied Mathematics and Computation, vol. 218, no. 17, pp. 8526-8536, 2012.

[22] X. Zhang and Q. Zhong, "Triple positive solutions for nonlocal fractional differential equations with singularities both on time and space variables," Applied Mathematics Letters, vol. 80, pp. 12-19, 2018.

[23] Y. Zou and G. He, "A fixed point theorem for systems of nonlinear operator equations and applications to (p1,p2)-Laplacian system," Mediterranean Journal of Mathematics, vol. 15, article no. 74, 2018.

[24] X. Tang, "Existence of solutions of four-point boundary value problems for fractional differential equations at resonance," Applied Mathematics and Computation, vol. 51, no. 1-2, pp. 145160, 2016.

[25] X. Tang, C. Yan, and Q. Liu, "Existence of solutions of two-point boundary value problems for fractional p-Laplace differential equations at resonance," Applied Mathematics and Computation, vol. 41, no. 1-2, pp. 119-131, 2013.

[26] M. Zuo, X. Hao, L. Liu, and Y. Cui, "Existence results for impulsive fractional integro-differential equation of mixed type with constant coefficient and antiperiodic boundary conditions," Boundary Value Problems, vol. 2017, article no. 161, 2017.

[27] Q. Sun and Y. Cui, "Solvability of $(k, n-k)$ conjugate boundary value problems with integral boundary conditions at resonance," Journal of Function Spaces, vol. 2016, Article ID 3454879 , 7 pages, 2016.

[28] Q. Sun and Y. Cui, "Existence results for (k,n-k) conjugate boundary-value problems with integral boundary conditions at resonance with dimkerL=2," Boundary Value Problems, article no. 29, 2017.

[29] T. Qi, Y. Liu, and Y. Zou, "Existence result for a class of coupled fractional differential systems with integral boundary value conditions," Journal of Nonlinear Sciences and Applications. JNSA, vol. 10, no. 7, pp. 4034-4045, 2017.

[30] Y. Wang, Y. Liu, and Y. Cui, "Multiple Solutions for a Nonlinear Fractional Boundary Value Problem via Critical Point Theory," Journal of Function Spaces, vol. 2017, Article ID 8548975, 8 pages, 2017.

[31] X. Zhang, L. Liu, Y. Wu, and Y. Cui, "Entire blow-up solutions for a quasilinear $\mathrm{p}$-Laplacian Schrodinger equation with a nonsquare diffusion term," Applied Mathematics Letters, vol. 74, pp. 85-93, 2017.

[32] Y. Cui, Q. Sun, and X. Su, "Monotone iterative technique for nonlinear boundary value problems of fractional order $\mathrm{p} \in(2,3]$," Advances in Difference Equations, vol. 2017, article no. 248, 2017.

[33] T. Jankowski, "Monotone iterative method for first-order differential equations at resonance," Applied Mathematics and Computation, vol. 233, pp. 20-28, 2014.

[34] C. Lu, C. Fu, and H. Yang, "Time-fractional generalized Boussinesq equation for Rossby solitary waves with dissipation effect in stratified fluid and conservation laws as well as exact solutions," Applied Matheamtics and Computation, vol. 327, pp. 104116, 2018.
[35] H. W. Yang, X. Chen, M. Guo, and Y. D. Chen, "A new ZK-BO equation for three-dimensional algebraic Rossby solitary waves and its solution as well as fission property," Nonlinear Dynamics, vol. 91, pp., pp. 2019-2032, 2018.

[36] A. A. Kilbas, H. M. Srivastava, and J. J. Trujillo, Theory and Applications of Fractional Differential Equations, New York, NY, USA, Elsevier, 2006.

[37] J. Mawhin, "Topological degree and boundary value problems for nonlinear differential equations," in Topological Methods for Ordinary Differential Equations, M. Furi and P. Zecca, Eds., vol. 1537 of Lecture Notes in Mathematics, pp. 74-142, Springer, Berlin, Germany, 1993. 


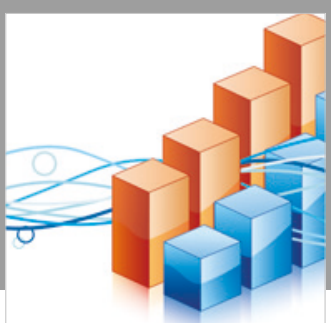

Advances in

Operations Research

\section{-n-m}
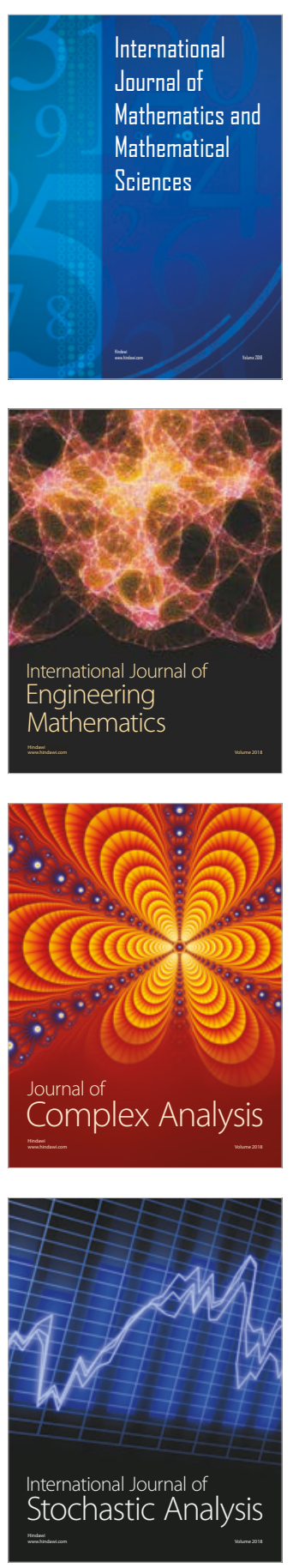
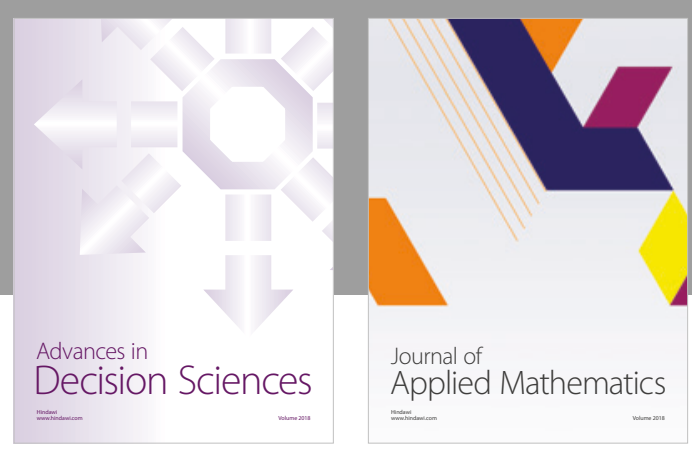

Journal of

Applied Mathematics
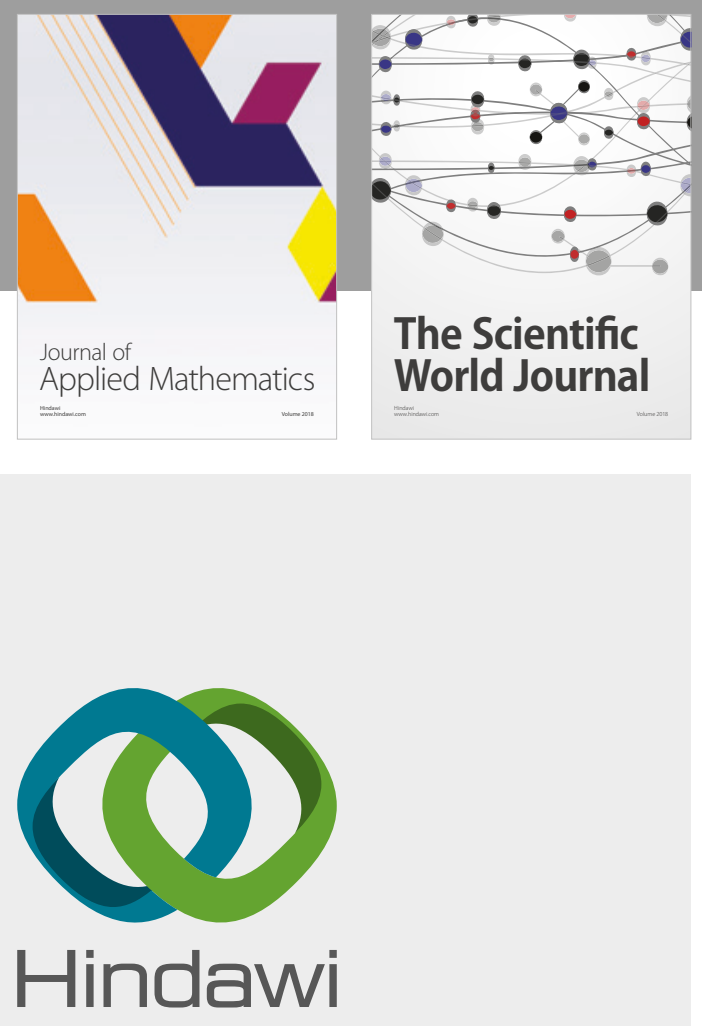

Submit your manuscripts at

www.hindawi.com

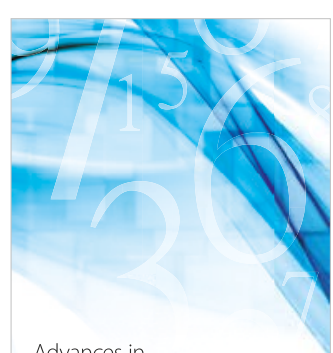

Advances in
Numerical Analysis
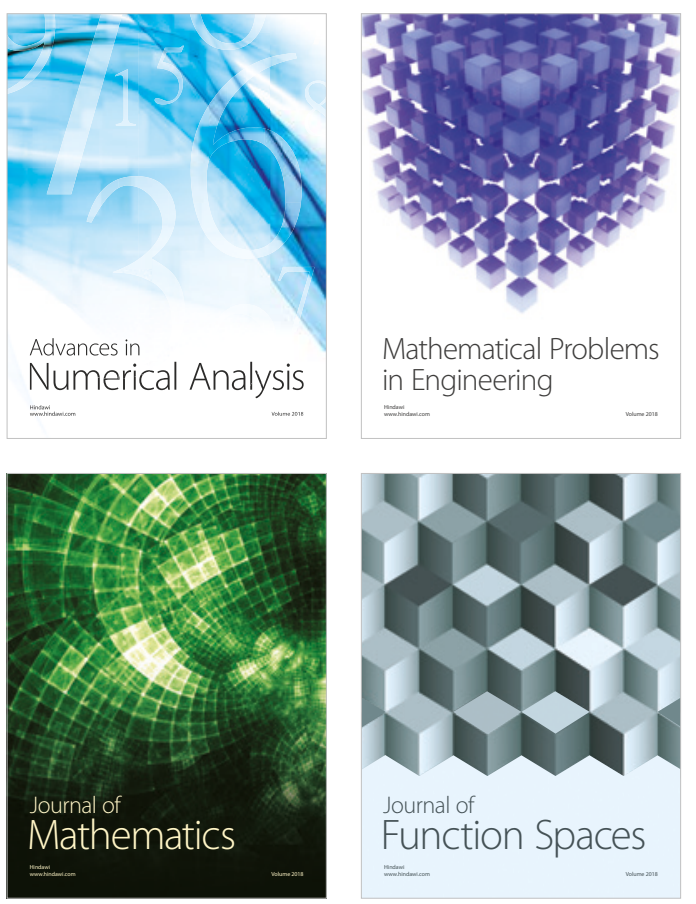

Mathematical Problems in Engineering

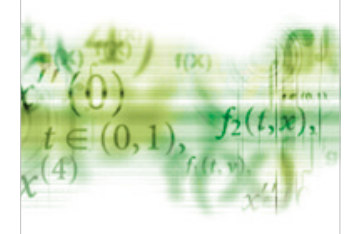

International Journal of

Differential Equations

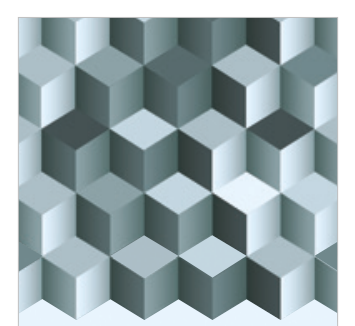

Journal of

Function Spaces

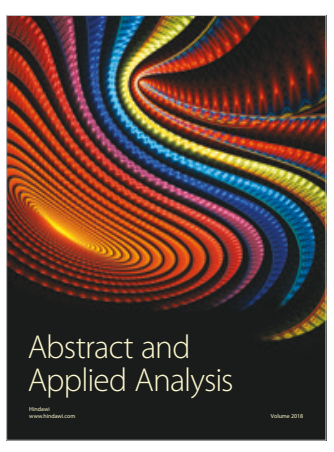

The Scientific

World Journal

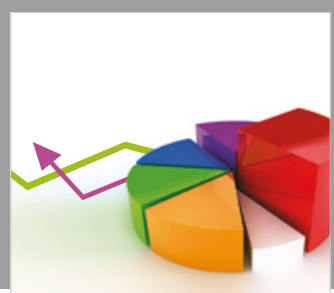

Journal of

Probability and Statistics
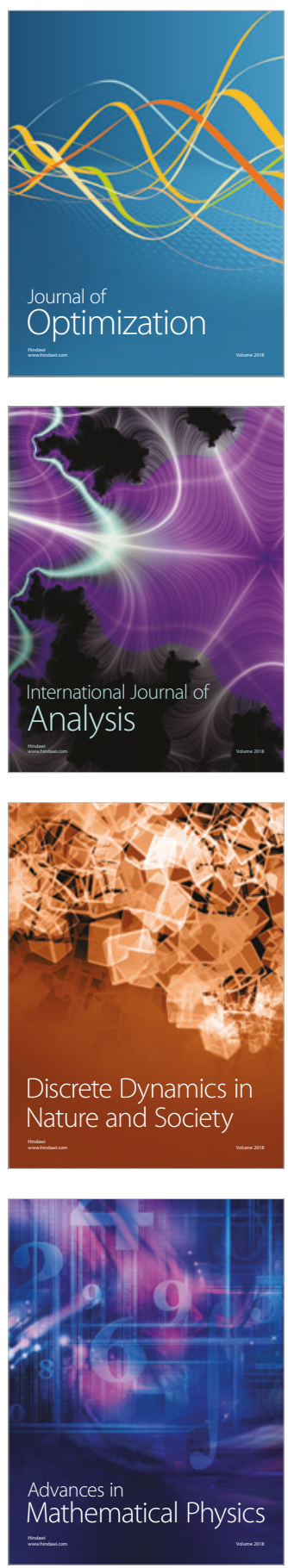